\title{
Growth and Characterization of Single Crystals of Alkali-Halides
}

\author{
Satyajit Kar \\ Department of Physics, Ramakrishna Mission Sikshanamandira, Belur Math, Howrah-711202, India
}

\begin{abstract}
A simple way to grow high quality single crystals of pure and mixed alkali halides synthesized from Czochralski-Kyropoulous method. The grown crystals are characterized by adopting usual practices along with a supporting experiment on electron injection to producing $\mathrm{F}$ - color center. The effect of doping concentration of $\mathrm{KBr}$ in stressed $\mathrm{KCl}$ matrix analyzed by estimating the activation energy. The indigenously built system with standard automation evolved satisfactory results.
\end{abstract}

Keywords: Czochralski - Kyropoulous method, Growth from melt, Color center, Electron Injection, Activation energy

\section{Introduction}

Decade long crystal growth was practiced as a new industry but an old subject. Various techniques are employed to grow perfect crystals of different materials involving costs in a completely different fashion. Real crystals are finite and must contain defects with the presence of having long-range order and this long-range order provides crystals their characteristic properties. Innumerable crystals have been grown by studying their inevitable defects [1-3] and thereby developed instrumentation accordingly.

Theory relating crystal radius to the time-variation of the force exerted on the pull-rod by the weight of the growing crystal and surface tension forces has been developed [4-6]. The nature of the relationship is investigated for different classes of materials and an anomalous dependence demonstrated for the case of materials, which expand on solidification and/or not completely wetted by their own melts. The consequences for automatic diameter control are considered.

In common with the other melt growth methods, crystal pulling is applicable only to materials, which melt congruently or near congruently, i.e., the compositions of the solid and the melt in equilibrium with it cannot differ much. However, crystal-pulling technique is usually faster than Bridgman growth so that less contamination occurs. Until reliable automation systems are developed, pulling requires more person-hour of labour. The capital cost of pulling is higher than the Bridgman technique and only part of this extra cost can be discounted by the faster growth rate. It may be mentioned that in Czochralski process, many impurities are lost from the melt on evaporation. The advantage of Kyropoulous process from the other one was that the segregation coefficient of the impurities allows noticing the first few layers on pulling of the crystal from the melt; hence, the central region of the grown single crystal is devoid of impurities largely.

Kyropoulous method is only attractive for a restricted range of materials destined for a limited range of uses. The method is widely used for alkali halides to make optical components for long wavelength applications. In the present work, we have applied the Czochralski-Kyropoulous (CZKyropoulous) technique to prepare alkali halide crystals, pure and mixed, like $\mathrm{KCl}, \mathrm{KBr}$, and $\mathrm{KCl}: \mathrm{KBr}$. Different parameters viz. temperature of solid-liquid interface, diameter, growth rate of the crystal, rotation of the molten mass, have been taken into consideration to develop an insight how they were interlinked with one another and the way the defects in the crystal from the stand point of instrumentation be minimized. Characterization through XRD will reveal the doping nature of the grown crystals. An experiment is also conducted to inject electron on to the grown crystals both pure and mixed varieties to assess the quality of growth along with the XRD studies.

\section{Experimental}

\subsection{Crystal Growth}

In the present the crystals are synthesized with the help of Czochralski-Kyropoulous method The system is designed with an aim to develop a dedicated crystal-growing unit, for crystals of melting point of wide range and of minimum mechanical faults as far as the machine is concerned. The system has a microprocessor-controlled furnace and a servo controlled rotating and pulling system (Fig.1).
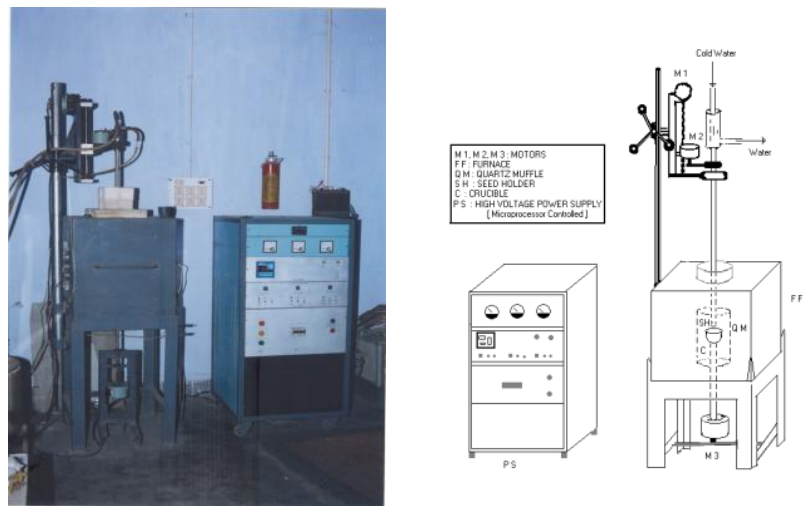

Figure 1: Typical Crystal Growth Unit

The designed furnace has a muffle and silicon carbide rods used as heating elements. The silicon carbide rods have an overall length of $55 \mathrm{~cm}$ and thickness $1.9 \mathrm{~cm}$. The hot zone 


\section{International Journal of Science and Research (IJSR) \\ ISSN (Online): 2319-7064}

Index Copernicus Value (2013): 6.14 | Impact Factor (2014): 5.611

consists of $15 \mathrm{~cm} \times 1.2 \mathrm{~cm}$ and nine resistances of value 1.3 $\Omega$ are used in three phases.

The furnace covers a total area of $55 \mathrm{~cm}$ (length) x $50 \mathrm{~cm} \mathrm{x}$ $51 \mathrm{~cm}$. The hot zone has a measure of $200 \mathrm{~cm} \times 20 \mathrm{~cm} \times 15$ $\mathrm{cm}$.

The heater power supply consists of SCR with manual and automatic control of power using PID based controller MPC - 500. A step down transformer 7.5 KVA is used to convert $400 \mathrm{~V} 50 \mathrm{~Hz}$ to $90 \mathrm{~V}$ through phase-to-phase conventional connections. Power controller is $1 \%$ to $99 \%$ proportional by SCR mounted on aluminium heat sink; a conventional chrome-alumel thermocouple senses the temperature of the heating elements. The output signal is compared with the set point in MPC - 500. The temperature control accuracy in the hot zone is $\pm 1^{0} \mathrm{C}$ and the temperature uniformity in the hot zone is $\pm 0.5^{0} \mathrm{C}$. The furnace temperature can be varied from room temperature to $1700^{\circ} \mathrm{C}$ at any desired rate and the furnace can be maintained at any temperature for any length of time.

The system can be programmed for the growth of different crystals simultaneously by fitting various programs under different programmed numbers (10 such program numbers are available simultaneously). For growing a particular crystal, a particular crystal a particular program can be made to operate manually or in automatic mode. Each program increases the sample temperature from the room temperature to a reasonable degree above the melting point of the sample where the powdered material is made sure to melt. Then the furnace temperature automatically (auto mode) drops to a value few degrees above the melting point when the crystal growth process can be started. Ten segments are available in a single program that makes ten different temperatures available at which the furnace can be maintained for any desired period. Such specialized care for maintenance of optimum temperature is very much beneficial since this ensures that the molten flux will solidify neither as the seed touches the melt nor the crystal will melt during pulling due to excess heat. Thus, the process allows forming the crystal of different diameters. The dedicated control of all the parameters viz. pulling rate, rotation speed both clockwise and anti- clockwise directions and the rotation of the molten mass in both the directions are maintained. Highly pure argon gas is inserted within the furnace through a platinum nozzle stainless steel capillary pipe in order to maintain the inert atmosphere within the furnace. Grown crystals $\mathrm{KCl}, \mathrm{KBr}$, and $\mathrm{KCl}: \mathrm{KBr}$ are photographed and is shown in Fig.2.

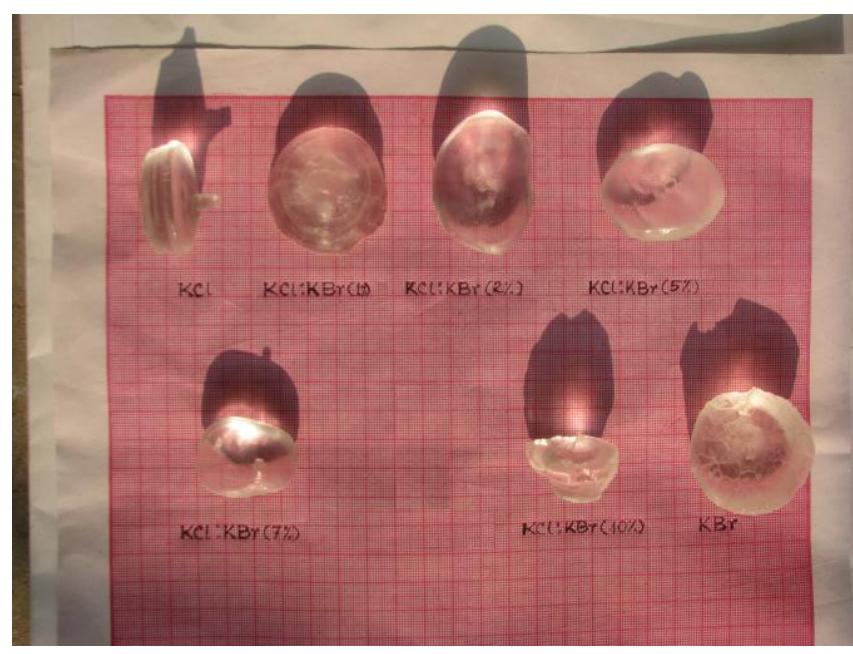

Figure 2: Photographs of some grown crystals.

Measuring in larger units, say some hundreds of atomic dimensions, growth face may be considered as flat one.

\subsection{Theoretical Interpretation of Crystal Growth}

During growth as the system is in equilibrium, it has a minimum free energy. If the system has a free energy $\Delta G$ above the minimum, it will try to reduce $\Delta G$. Then we can consider $\Delta G$ as the driving force for the growth of crystal. If other relevant factors remain the same, rate of growth of crystal will be an increasing function of the driving force $(\Delta G)$. In reality, since the absolute dislocation free crystal is not possible to grow, the ideal condition of $\Delta G=$ 0 is not realizable in practice. However, it is a desirable state.

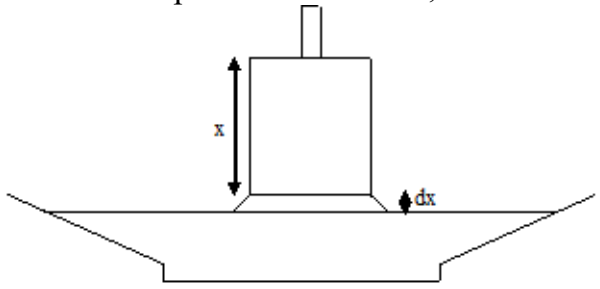

Figure 3: Interface of grown and to be grown crystal.

Let us draw a diagram (Fig.3) in which we have considered the length of the grown crystal be $x$ and $d x$ is the small element in the interface of grown and to be grown process. If $\frac{d x}{d t}$ be the growth rate at the moment when crystal of length $x$ has already been grown (shown in Fig. 3), the expected growth equation can be written as,

$$
\rho \frac{d x}{d t} \alpha L=\frac{K\left(\theta_{f}-\theta_{0}\right) \alpha}{x}+\text { Heat loss }
$$

where $\rho$ is the density of the solid material, $\alpha$ is the crosssectional area of the grown crystal, $L$ is the latent of solidification, $\mathrm{K}$ is the thermal conductivity, $\theta_{f}$ is the temperature of the solid-liquid interface, $\theta_{0}$ is the temperature of the seed and grown crystal interface, i.e., at $x=0$. 


\section{International Journal of Science and Research (IJSR) \\ ISSN (Online): 2319-7064}

Index Copernicus Value (2013): 6.14 | Impact Factor (2014): 5.611

In practice, heat loss is very small and neglected; the equation takes the form,

$$
\begin{aligned}
& \rho L \int_{0}^{l} x d x=K\left(\theta_{f}-\theta_{0}\right) \int_{0}^{t} d t \\
\text { or, } \quad & \frac{l^{2}}{2}=\frac{K}{\rho L}\left(\theta_{f}-\theta_{0}\right) t \\
\text { or, } \quad l & \left.l \frac{2 K}{\rho L}\left(\theta_{f}-\theta_{0}\right) t\right]^{\frac{1}{2}}=f(t)
\end{aligned}
$$

Experimental evidences show that size (in diameter) of the crystal [7] is the function of rotation of the seed $(\omega)$, its temperature $(\mathrm{T})$ while $\mathrm{T}$ in turn the function of the rate of flow of water $\left(\frac{d m}{d t}\right)$ in the cooling system.

In the present experiment, the rate of flow of water $\left(\frac{d m}{d t}\right)$ in the cooling jacket has been kept constant. Temperature of the flowing water will increase, resulting in a decrease in the diameter of the grown crystal. Variations in size of the grown crystal and corresponding change in temperature of the coolant with time of growth have been studied for the different alkali halides. A typical result of a pure $\mathrm{KBr}$ crystal is presented in table- I, and the corresponding figures are shown in Fig.4.

Table 1: Change of Temperature and the crystal diameter with time.

\begin{tabular}{|c|c|c|}
\hline Time ( mins.) & Diameter $(\mathbf{c m})$ & Temperature $\left.\mathbf{~}^{\mathbf{0}} \mathbf{C}\right)$ \\
0 & 2.45 & 31.4 \\
10 & 2.45 & 31.5 \\
20 & 2.60 & 31.7 \\
30 & 2.87 & 31.8 \\
40 & 2.78 & 32.0 \\
50 & 2.70 & 32.2 \\
60 & 2.63 & 32.3 \\
70 & 2.54 & 32.5 \\
80 & 2.40 & 32.6 \\
90 & 2.32 & 32.9 \\
100 & 2.25 & 33.0 \\
110 & 2.10 & 33.0 \\
\hline
\end{tabular}

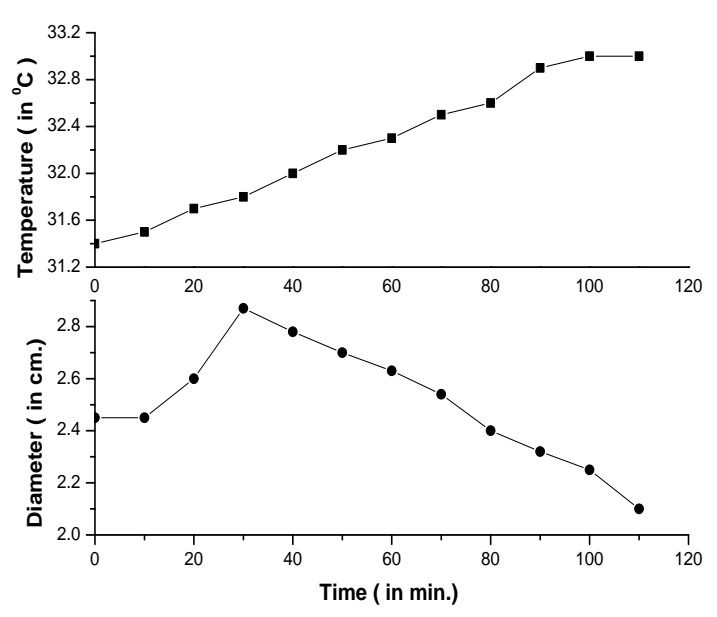

Figure 4: Rate of change of temperature; and the variation of the crystal diameter with time.
It is clear from Fig.4 that the slopes are of opposite sign, which is obvious. In the $\mathrm{CZ}$ system, many impurities are lost from the melt by evaporation. Most of the impurities have segregation coefficients less than unity, which means that at a growth face they are rejected [8]. Thus, in melt growth we have a situation that when the melts are not pure, there is a high concentration of impurities near the grown face. Hence, we have restricted the free melt surface in the present system of crystal growth.

\subsection{Characterization}

Crystal specimen of $\mathrm{KCl}, \mathrm{KBr}$, and $\mathrm{KCl}: \mathrm{KBr}(1 \%, 2 \%, 5 \%$, $10 \%$ ) grown indigenously are cleaved in $<100>$ direction and polished by wet and dry chamois leather. The specimen is mounted (Fig.5) between a flat platinum anode and a brass pointed cathode and housed in an electronically controlled furnace whose temperature is measured with the help of chrome alum thermocouple.

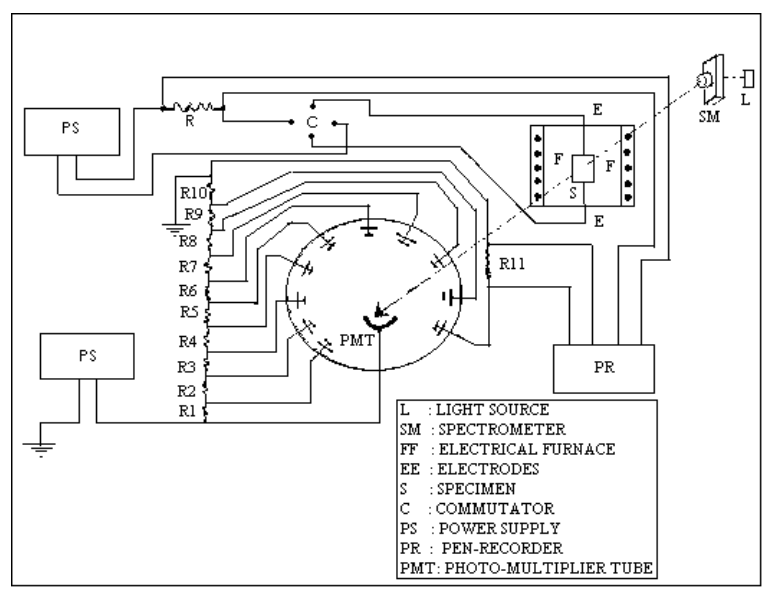

Figure 5: General Experimental Arrangement.

On achieving a certain temperature of the specimen, fixed electric field of $800 \mathrm{Vcm}^{-1}$ is applied between the heterogeneous electrodes. The variation of injection current with temperature is recorded with the help of a Bausch and Lomb series 5000 strip chart dual pen recorder. The injection current is restricted within ionic zone [9-14].

To characterize the grown crystals the following steps have been imparted carefully. Firstly, X- ray diffraction (XRD) pattern of each specimen was obtained with the help of BRUKER -AXS -D8 Advance using $\mathrm{Cu}-\mathrm{K}_{\alpha}$ radiation of monochromatic wavelength 1.5406 A. Fig. 6 shows the typical XRD pattern. The shift of the diffraction angles in the $\mathrm{XRD}$ is essentially due to the incorporation of $\mathrm{KBr}$ in $\mathrm{KCl}$. In order to check the appropriateness of the grown crystals and the nature of the shift in the intensity pattern we follow a simple experiment.

\section{Results and Discussion}

The XRD patterns (Fig.6) for pure $\mathrm{KBr}$ and Pure $\mathrm{KCl}$ to the extreme ends depicts that the generic nature of their formation in the planes e.g., $\mathrm{KCl}$ is on the right while $\mathrm{KBr}$ falls on the left flank. On doping $\mathrm{KBr}$ in $\mathrm{KCl}$ matrix, the shift 


\section{International Journal of Science and Research (IJSR) \\ ISSN (Online): 2319-7064}

Index Copernicus Value (2013): 6.14 | Impact Factor (2014): 5.611

towards the $\mathrm{KBr}$ side is distinctly observed. This is one of the ways to check the appropriate formation of the crystals.

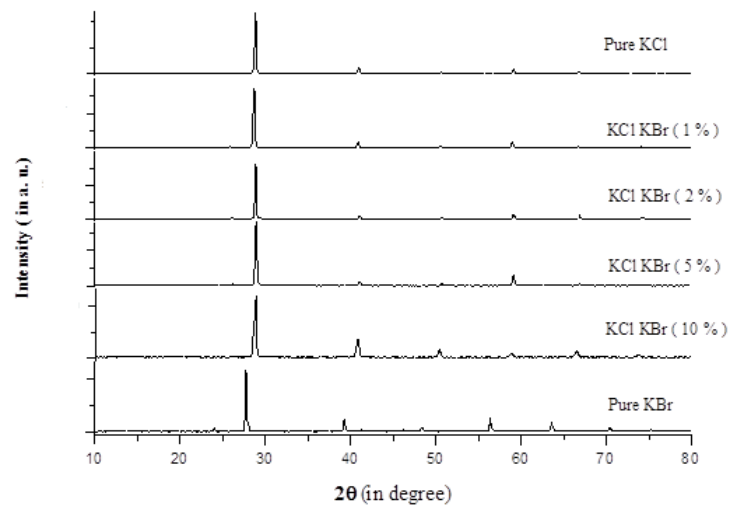

Figure 6: XRD pattern of the grown crystals showing the shift in the peak positions due to incorporation of $\mathrm{KBr}$ in $\mathrm{KCl}$ matrix.

The shifting of the diffraction angles in the XRD pattern (Fig.6) due to the incorporation of $\mathrm{KBr}$ in $\mathrm{KCl}$ matrix is confirmed with the electron injection experiment by measuring the sequential change in activation energy values.

The characteristic growth of ionic current with temperature for all the specimens under constant electric field is shown in Fig.7. The nature of growth pattern has a tendency to proceed towards $\mathrm{KBr}$ crystal when percentage of doping is increased. Current growth due to this solid electrolysis through heterogeneous contacts at high temperature resemble somewhat with the injection from a thermionic cathode into vacuum. Writing the current density as a function of temperature as we generally do with the thermionic cathode emission viz.,

$$
j=A e^{-\left(\frac{W_{0}}{k T}\right)}
$$

From the plot of $\ln j$ versus $\frac{1}{T}$ (Fig. 8), the activation energy $\left(W_{0}\right)$ values have been obtained, shown in the table-II.

Table 2: Estimated activation energy values for $\mathrm{KCl}, \mathrm{KBr}$ and $\mathrm{KCl}: \mathrm{KBr}(1,2,5,10$ mole $\% \mathrm{KBr})$.

\begin{tabular}{|c|c|}
\hline Crystal & Activation Energy (in eV) \\
$\mathrm{KCl}($ pure $)$ & 0.978 \\
$\mathrm{KCl}: \mathrm{KBr}(1 \%)$ & 1.005 \\
$\mathrm{KCl}: \mathrm{KBr}(2 \%)$ & 1.036 \\
$\mathrm{KCl}: \mathrm{KBr}(5 \%)$ & 1.046 \\
$\mathrm{KCl}: \mathrm{KBr}(10 \%)$ & 1.054 \\
$\mathrm{KBr}($ Pure $)$ & 1.061 \\
\hline
\end{tabular}

Actually, in ionic solid like alkali halides the electric current under electrolysis is due to thermally enhanced motion of ions. Upon electrolysis the anions reaches the cathode whereas cations to the anode. The heterogeneous contact creates the non-uniform fields, which in turn accelerate to form ohmic contact to cross over ionic zone; a space charge limited one called the second zone or the coloration to commence [15-20].

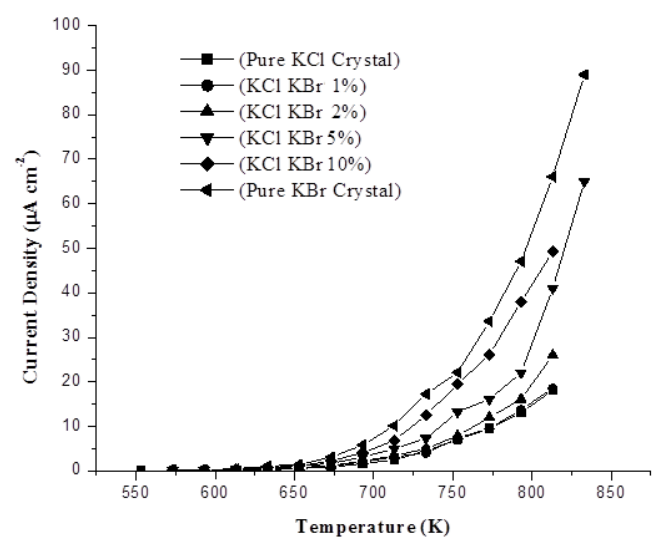

Figure 7: Ionic current growth under injection field of 800 $\mathrm{Vcm}^{-1}$ at various temperatures.

In the present experiment, the coloration under space charge limited condition will prevail only after the formation of appropriate ohmic contact in the heterogeneous contacting geometry. The necessary activation energy required to form this appropriate contact can be seen in table-II. The change in the activation energy with the addition of doping material has an impact on to the flow of electron to form color center in the second zone (SCL).

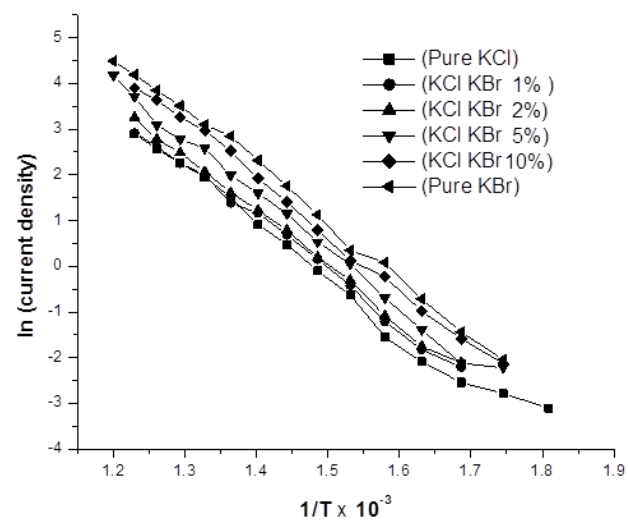

Figure 8: Logarithm of current density $(\ln J)$ as a function of reciprocal of temperature under injection, field $800 \mathrm{Vcm}^{-1}$.

Moreover, the injection on to the crystals generates the color center of the kind characteristics of either $\mathrm{KCl}$ (magenta) or to that of $\mathrm{KBr}$ (green). A defective or possessing excess strained crystal does not show the characteristic colors, rather a dirty blackish color. Thus, we conclude that the grown crystals are comparable with those grown in high quality instruments. In other words, one can support the melt growth of crystals and can have the flexibility by controlling the experimental parameters as per requirements of different materials.

\section{Conclusions}

A good quality single crystal of pure and mixed alkali halides synthesized from Czochralski-Kyropoulous method. The grown crystals are characterized by adopting usual practices along with a supporting experiment on electron injection to producing F- color center. Injection experiment has been performed to extract injection and photocurrents at constant 


\section{International Journal of Science and Research (IJSR) \\ ISSN (Online): 2319-7064 \\ Index Copernicus Value (2013): 6.14 | Impact Factor (2014): 5.611}

field stress under different temperature ambient. The variation of the conductivity of injecting carriers with the color centers yielded activation energy value, which is found to have a threshold limit of doping with $\mathrm{KBr}$ in $\mathrm{KCl}$ matrix. Thus the change in conductivity has been studied with transparency that is being quantified by optical density. From the same condition of trapping, conductivities for different temperatures have been extrapolated. The temperature dependent O.D. as well as conductivity will provide a signature to semi-conducting behavior of the specimen which will confirm the phase transition because originally the specimen at room temperature is an insulator.

Mixed crystals like $\mathrm{KCl}: \mathrm{KBr}$ of different mole \% have been treated under same thermal and field stresses. The effects of mole \% doping on conductivity and the overall physical behavior have been discussed. The effect of doping concentration of $\mathrm{KBr}$ in stressed $\mathrm{KCl}$ matrix analyzed by estimating the activation energy. The indigenously built system with standard automation evolved satisfactory results.

\section{Acknowledgements}

The author wishes to thank Dr. A. K. Maiti of Physics Department, Bidhannagar Govt. College and Prof. K Goswami of Physics Department, Jadavpur University for helping during growth and characterization.

\section{References}

[1] K. C. Kao and W. Huang, Electrical transport in solids, Vol. 14, Pergamon Press (1981) p 73 - 90;

[2] N. F. Mott and R. W. Gurney : Electronic Processes in Ionic Crystals, Dover publications Inc., New York (1964), second edition, Ch. 4;

[3] M. A. Lampert and P. Mark : Current Injection in solids, Academic Press, New York (1970), Ch. 5-9;

[4] B.R.Pamplin; Crystal Growth ( $2^{\text {nd }}$.Edition), Pergamon Press (1980).

[5] J.C. Brice; Crystal Growth Processes, Blackie, and Son Ltd., (1986)109.

[6] W.Bradsley et al; J.Crystal Growth, 40 (1977) 13.

[7] M. T. Montojo, F. Jaque and C. Sanchez, J. Phys. Chem. Solids, 38, (1977) 657;

[8] G.A Andreev, G.B Semuskin, \& A.N.Tsikin; Soviet Physics Solid State, 9 (1968) 2564.

[9] G.A Andreev, G.B Semuskin, \& A.N.Tsikin; Soviet Physics Solid State, 9 (1968) 2564.

[10] J.J.O'Dwyer; The theory of Electrical Conduction and breakdown in solid dielectrics, Clarendon Press, Oxford (1973).

[11]A.K.Lahiri, A.K.Maiti \& K.Goswami; Solid State Comm., 65, 5 (1988) 377.

[12] A.K.Maiti, K.Goswami, S. Choudhuri, and A.Choudhury; Electro chemical Soc. 128, No.9 (1981) 1995.

[13] S. Kar, A. K. Maiti, S. Bandyopadhyay, K. K. Ray and K. Goswami, FIZIKA A 11 (2002) 3, p. 113-120;

[14] M. Sengupta, A. K. Maiti, and K. Goswami : FIZIKA A 2, (1993) 3;
[15] J. H. Schulman \& W. D. Compton : Color Centers in Solids, Pergamon Press, Oxford, 1962, Ch. 1, 3;

[16] A.K.Lahiri, A.K.Maiti \& K.Goswami; Solid State Comm., 65, 5 (1988) 377.

[17] A.K.Maiti, K.Goswami, S. Choudhuri, and A.Choudhury; Electro chemical Soc. 128, No.9 (1981) 1995.

[18] S. Kar, A. K. Maiti, M. Sengupta and K. Goswami, FIZIKA A, 9, (2000) 4, p. 159-168;

[19] S. Kar, S. Bandyopadhyay, K.K. Ray, A.K. Maiti, A.K. Lahiri, K. Goswami: Journal of Crystal Growth 261 (2004) 533-538;

[20] S. Kar, A.K. Maiti S. Bandyopadhyay, K.K. Ray, A.K. Lahiri, K. Goswami: FIZIKA A 13 (2004) 2, 65-76.

\section{Author Profile}

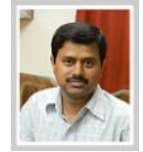

Dr. Satyajit Kar, M.Sc.(Physics), Ph. D. (2004) from Jadavpur University, Kolkata, INDIA. He is working as an Assistant Professor in Physics at Ramakrishna Mission Sikshanamandira, Belur Math, Howrah, India. 